(1)

CrossMark

\title{
The time-controlled adaptive ventilation protocol: mechanistic approach to reducing ventilator-induced lung injury
}

\author{
Michaela Kollisch-Singule (10), Penny Andrews², Joshua Satalin', Louis A. Gatto ${ }^{1,3}$, \\ Gary F. Nieman ${ }^{1}$ and Nader M. Habashi ${ }^{2}$
}

Affiliations: ${ }^{1}$ Dept of Surgery, SUNY Upstate Medical University, Syracuse, NY, USA. ${ }^{2}$ Dept of Trauma Critical Care Medicine, R Adams Cowley Shock Trauma Center, University of Maryland School of Medicine, Baltimore, MD, USA. ${ }^{3}$ Dept of Biological Sciences, SUNY Cortland, Cortland, NY, USA.

Correspondence: Joshua Satalin, SUNY Upstate Medical University, 750 E. Adams Street, Syracuse, NY 13210. E-mail: satalinjaupstate.edu

@ERSpublications

Randomised controlled trials are needed to test the efficacy of the TCAV protocol further; however, experimental evidence demonstrates that TCAV may be effective in preventing VILI and ARDS by a pleiotropy of mechanisms http://ow.ly/uT8J30nSGeN

Cite this article as: Kollisch-Singule $\mathrm{M}$, Andrews $\mathrm{P}$, Satalin J, et al. The time-controlled adaptive ventilation protocol: mechanistic approach to reducing ventilator-induced lung injury. Eur Respir Rev 2019; 28: 180126 [https://doi.org/10.1183/16000617.0126-2018].

ABSTRACT Airway pressure release ventilation (APRV) is a ventilator mode that has previously been considered a rescue mode, but has gained acceptance as a primary mode of ventilation. In clinical series and experimental animal models of extrapulmonary acute respiratory distress syndrome (ARDS), the early application of APRV was able to prevent the development of ARDS. Recent experimental evidence has suggested mechanisms by which APRV, using the time-controlled adaptive ventilation (TCAV) protocol, may reduce lung injury, including: 1) an improvement in alveolar recruitment and homogeneity; 2) reduction in alveolar and alveolar duct micro-strain and stress-risers; 3) reduction in alveolar tidal volumes; and 4) recruitment of the chest wall by combating increased intra-abdominal pressure. This review examines these studies and discusses our current understanding of the pleiotropic mechanisms by which TCAV protects the lung. APRV set according to the TCAV protocol has been misunderstood and this review serves to highlight the various protective physiological and mechanical effects it has on the lung, so that its clinical application may be broadened.

\section{Introduction}

Acute respiratory distress syndrome (ARDS) has an estimated annual incidence of 141500 cases requiring $>2.5$ million hospital days in the United States alone [1] and a hospital mortality of $>40 \%$ [2]. The ventilator itself, although necessary to support patients with lung injury, is known to propagate lung injury, often progressing patients into ARDS [3]. Once established, ARDS can further evolve from mild to moderate to severe ARDS, with a corresponding increase in mortality [4]. In order to prevent this ventilator-induced lung injury (VILI), protective mechanical ventilation strategies have been advocated [5], most notably the low tidal volume strategy from the ARDSnet trial in 2000 [6]. Despite the development of these protective mechanical ventilation practices, the mortality associated with ARDS remains unacceptably high [4].

Airway pressure release ventilation (APRV) is considered a rescue ventilation mode by some clinicians [7], but has been advocated as a pre-emptive, protective mechanical ventilation strategy based on durable

Provenance: Submitted article, peer reviewed.

Received: Dec 272018 | Accepted after revision: Feb 162019

Copyright CERS 2019. This article is open access and distributed under the terms of the Creative Commons Attribution Non-Commercial Licence 4.0. 
clinical experience [8] and experimental work [9-12]. It is important to note that APRV, as with all modes, can be set in a multitude of ways and thus we must analyse the protocol using APRV in order to compare efficacy in published studies. In order to clarify the confusion in nomenclature [13], one well-accepted protocol of setting APRV was recently defined as time-controlled adaptive ventilation (TCAV) [14], and refers to the protocol first described by HABASHI [15], with an extended time at the plateau pressure, creating a continuous positive airway pressure (CPAP) phase, and a brief release in CPAP, creating a release phase. The release phase augments carbon dioxide removal and the resultant slope of the expiratory flow-time curve from the passive pressure release provides continuous feedback of changes in respiratory system elastance [15-18]. Although the term TCAV has only recently been defined, APRV set using this method has been in effect for over a decade [15] and studies using this method (although not explicitly named as TCAV) are reviewed with the objective of demonstrating how the physiological effects of APRV might translate into clinical usefulness.

It has been suggested recently that the importance of determining the impact of a given ventilator strategy on the lung micro-environment is often overlooked, favouring instead macroventilatory parameters such as tidal volume $(V \mathrm{~T})$ and plateau pressure $(P$ plat $)$ to dictate management [19]. In a prospective and retrospective clinical review [8, 20] and multiple experimental animal studies [9-12], TCAV has demonstrated improvements in oxygenation and lung function, with the ability to prevent ARDS, with a matching reduction in micro-anatomical injury [11,21]. Experimental data have demonstrated that targeting the microventilatory environment with TCAV improves alveolar recruitment and stability, maximising alveolar surface area without causing overdistension [14, 22, 23] and improves oxygenation [9, 11, 24]. In addition, it stabilises the alveoli, reducing alveolar microstrain [22] and preserving surfactant protein [9, 11]. The pleiotropic mechanisms by which TCAV may be effective in preventing lung injury are herein reviewed.

\section{Alveolar recruitment}

The pressure-time integral (PTI) describes the degree of pressure the respiratory system is exposed to over a given ventilator cycle [11]. Due to the ability to finely regulate the time in milliseconds at the $P$ plat (CPAP phase) and the time during the release phase, APRV is able to precisely and independently (impartial controls for Pplat and release phase) regulate the PTI according to the physiology of the patient's respiratory system $[11,15-18,25,26]$. Furthermore, the extended time during Pplat gives TCAV a significantly greater PTI than the standard of care low VT ventilation (LVT; figure 1) [11, 22]. The higher

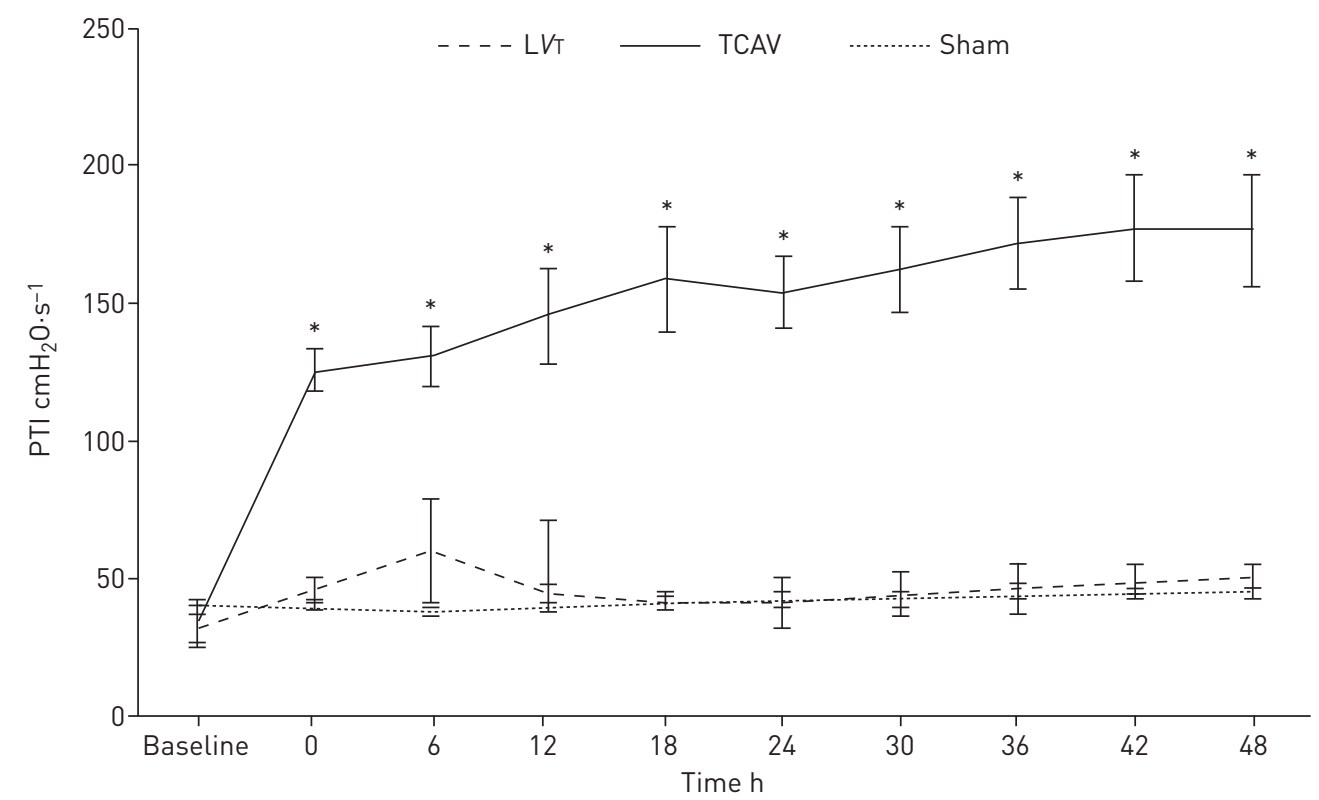

FIGURE 1 The pressure-time integral (PTI) measures the degree of pressure to which the respiratory system is exposed over a given ventilator cycle. In a porcine extrapulmonary acute respiratory distress syndrome model, the time-controlled adaptive ventilation (TCAV) protocol was compared with low tidal volume (LVT) and a sham group with no injury (also mechanically ventilated according to the LVT protocol). With an extended time at the set upper pressure (Phigh), airway pressure release ventilation set by the TCAV protocol has a significantly higher PTI as compared with LVT or the sham group. The higher PTI allows for additional time at inspiration to maximally recruit alveoli and a brief expiratory duration prevents alveolar collapse. Reproduced and modified from [11] with permission. 
PTI provides the entire respiratory system with additional time to equilibrate the delivered inspiratory pressure, thus allowing for distribution of pressure to the chest wall and to recruiting the distal airspaces rather than being confined to only the more proximal airways [26].

Using in vivo microscopy to visualise the alveoli of rat lungs with a direct lung injury involving detergent-induced surfactant deactivation, TCAV recruited the greatest number of alveoli and improved alveolar surface area compared with conventional LVT ( $\mathrm{L} V \mathrm{~T}$ with tidal volumes of $6 \mathrm{cc} \cdot \mathrm{kg}^{-1}$ ) despite positive end-expiratory pressure (PEEP) levels up to $24 \mathrm{cmH}_{2} \mathrm{O}$ [22]. In animal studies involving an indirect lung injury, TCAV has demonstrated an improvement in oxygenation with a reduction in ARDS incidence as compared with $\mathrm{L} V \mathrm{~T}$ as well as high $V \mathrm{~T}\left(\mathrm{H} V \mathrm{~T}\right.$; $V \mathrm{~T}$ of $10 \mathrm{cc} \cdot \mathrm{kg}^{-1}$ and PEEP $\left.5 \mathrm{cmH}_{2} \mathrm{O}\right)$. In these studies, pigs with early mortality on the TCAV protocol were often still being oxygenated with an inspiratory oxygen fraction $\left(\mathrm{FIO}_{2}\right)$ of $21 \%$ and death was due to haemodynamic collapse from sepsis and not from lung injury $[9,11]$. This improvement in oxygenation is in part due to alveolar recruitment from the increased PTI $[11,22,26]$. However, alveolar recruitment itself may not be sufficient to maintain oxygenation and prevent lung injury without sustained time-dependent alveolar stabilisation, preventing recurring collapse and cyclic recruitment/derecruitment (R/D), ultimately inducing a durable homogenous lung that decreases stress-risers [27-29]. The homogeneity induced with the extended PTI at Pplat permits persistent alveolar recruitment with less energy transfer as compared to PEEP recruitment methods [30].

\section{Alveolar stability}

Flooding of alveoli with proteinaceous oedema leads to surfactant deactivation [3]. Surfactant is necessary to prop open the alveoli and prevent atelectrauma with expiration, and its absence leads to repetitive $\mathrm{R} / \mathrm{D}$-induced stress-risers. Injurious mechanical ventilation strategies can initiate or propagate this alveolar $\mathrm{R} / \mathrm{D}$, further wearing down surfactant. Surfactant inactivation leads to an increase in alveolar surface tension, potentially increasing microvascular transmural pressure which, combined with increased pulmonary microvascular permeability, causes alveolar oedema to accrue, further deactivating surfactant [31-33]. Thus this triad of alveolar oedema, instability and surfactant deactivation can propagate (figure 2) [9], particularly when coupled with injurious mechanical ventilation. A protective mechanical ventilation strategy must be considered to prevent all three elements of the triad in order to reduce VILI.

In order to test the physiological impact of ventilatory strategies on this triad, TCAV was compared with HVT [9] and LVT [11] in an extrapulmonary ARDS model of sepsis plus ischaemia/reperfusion. In these studies, TCAV reduced alveolar oedema as demonstrated by a reduction in alveolar fibrinous exudates [9-12], bronchoalveolar lavage fluid (BALF) protein $[9,10,12]$ and lung wet/dry weight $[9,11]$. In addition, TCAV demonstrated preservation of surfactant protein A concentrations as compared with $\mathrm{H} V \mathrm{~T}$ and $\mathrm{LVT}[9,11,12,34]$ and a preservation of lung architecture as compared with both HVT and LVT [9, $11,12]$. In rat studies of extrapulmonary ARDS, the TCAV group was found to have no lung oedema despite a higher volume of intravascular fluids infused [14]. In a supplemental study utilising in vivo microscopy, it was demonstrated that TCAV reduced alveolar R/D, suggesting an increase in alveolar stability [22]. Thus, TCAV is able to block all components of this pathological tetrad of alveolar oedema, instability and surfactant deactivation, promoting alveolar stability and homogeneity, making it an ideal protective mechanical ventilation strategy to reduce stress-risers.

\section{Biotrauma}

Injurious mechanical ventilation may harm the lung, causing release of inflammatory mediators and biotrauma [35]. This lung-derived inflammation, if left unopposed, may lead to overamplification of the inflammatory cascade leading to both a local and systemic inflammatory response $[35,36]$. This systemic inflammatory response may cause additional organ injury outside the lung with mechanical ventilation being linked to subsequent small intestine and kidney epithelial cell apoptosis [37], greater risk of developing septic syndrome [38] and increased haemodynamic instability and mortality [39]. Protective mechanical ventilation focused on preventing end-expiratory collapse and minimising lung strain has been linked to a reduction in biotrauma [40]. Thus, protective mechanical ventilation strategies may be able to reduce not only regional lung inflammation but the systemic inflammatory response syndrome and resultant multiple organ dysfunction [41]. APRV using the TCAV protocol has been shown to significantly lower BALF interleukin (IL)-8 and tumour necrosis factor- $\alpha$ compared with LVT and high-frequency oscillatory ventilation [42], as well as decreased IL-6 levels compared with HVT [9] and LVT [11], indicating that the lung is being exposed to a less injurious ventilation strategy and therefore may also predict a reduction in downstream inflammation and organ injury [37, 43].

In a study by SILVA et al. [14], rats were randomised to a pulmonary or extrapulmonary ARDS injury with either Escherichia coli pneumonia or E. coli peritoneal sepsis, respectively. Diffuse alveolar damage was decreased in both extrapulmonary and pulmonary ARDS groups in the TCAV group compared with the 

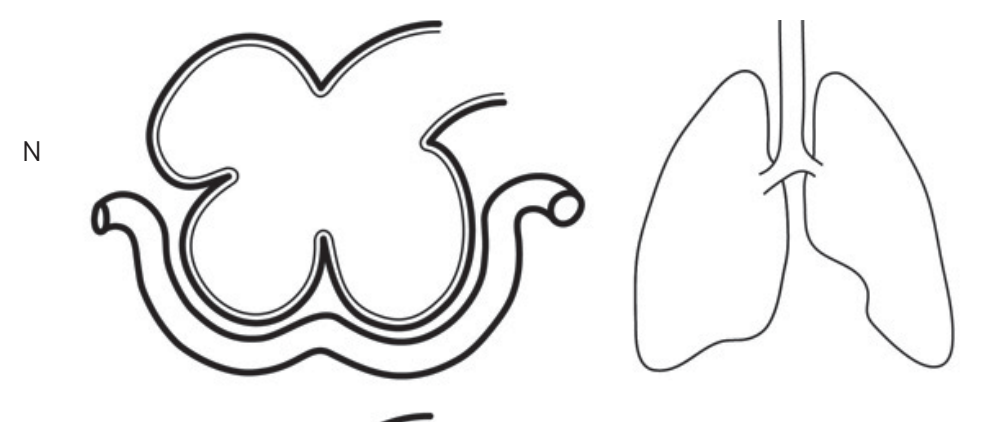

Clinical parameters

$\mathrm{P} / \mathrm{F}<200$

No

Infiltrated radiograph No

Refractory hypoxaemia No
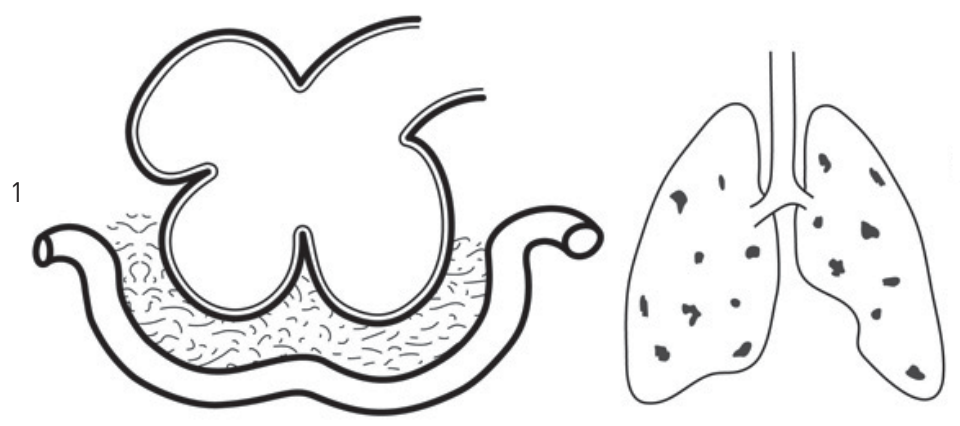

Clinical parameters

$\mathrm{P} / \mathrm{F}<200$

No

Infiltrated radiograph No

Refractory hypoxaemia No
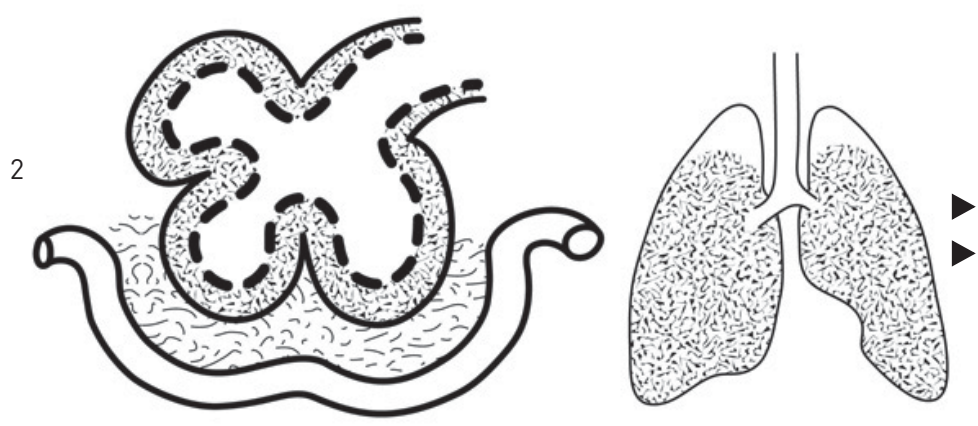

Clinical parameters

\section{$P / F<200$}

Yes

Infiltrated radiograph Yes Refractory hypoxaemia No
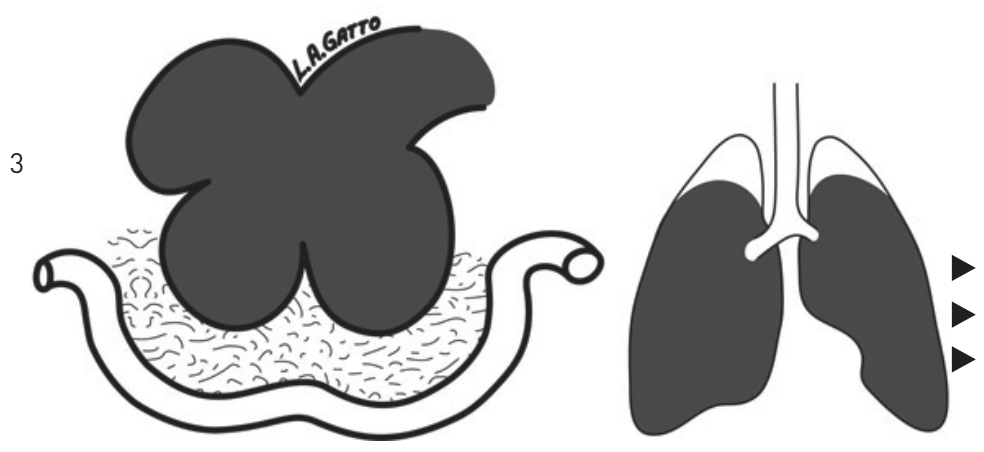

Clinical parameters

$P / F<200$

Infiltrated radiograph Yes

Refractory hypoxaemia Yes

FIGURE 2 Pathogenesis of acute respiratory distress syndrome (ARDS); the left column represents the alveolar complex coupled with the interstitial space and capillary; the right column represents the lung. $\mathrm{N}$ : normal homogenous alveoli; 1: occult ARDS with interstitial oedema, but no alveolar oedema or significant clinical symptoms; 2: pseudo-ARDS with interstitial oedema and initiation of alveolar oedema with surfactant deactivation, alveolar instability and clinical hypoxaemia; 3: fulminant-ARDS with interstitial and alveolar oedema, surfactant deactivation and refractory hypoxaemia. The triad of alveolar oedema, instability and surfactant deactivation can propagate from one stage to the next with ventilator-induced lung injury, but can be reversed with appropriately applied mechanical ventilation settings. Reproduced and modified from [9] with permission.

LVT group. In the pulmonary ARDS groups, the alveolar-capillary membrane was found to be the structure most affected by the injury model. LVT upregulated amphiregulin expression, whereas TCAV did not, suggesting that the TCAV protocol did not overstretch the alveoli (despite a higher Pplat and VT) [14]. In support of this, in a porcine extrapulmonary model of ARDS, E-cadherin expression was decreased in the LVT group compared with TCAV $[10,11]$, suggesting a relative loss of respiratory epithelium integrity and increased paracellular permeability in the LVT group [44]. TCAV has been found to increase vascular 
cell adhesion molecule-1 expression compared with $\mathrm{LVT}$, suggesting that the mechanical ventilation strategy was able to preserve endothelial integrity. In pulmonary ARDS, LVT has demonstrated increased matrix metalloproteinase-9 expression as compared with TCAV and with unventilated control rats, suggesting a decreased degradation of the extracellular matrix in TCAV [14].

\section{Chest wall mechanics}

TCAV does not target $V \mathrm{~T}$, but rather advocates for utilising the release phase (TLow) and the resulting slope of the expiratory flow curve as real-time feedback of changes in respiratory system elastance. A TLow set to terminate at $75 \%$ of the peak expiratory flow rate preserves a low alveolar $V \mathrm{~T}$ despite changes in respiratory system elastance or whole-lung $V \mathrm{~T}$ [22]. A patient with poor lung compliance (or increased elastance) on volume control ventilation targeting $6 \mathrm{cc} \cdot \mathrm{kg}^{-1}$ when transitioned to APRV with the TLow set to terminate the flow rate at $75 \%$ of the peak expiratory flow typically results in an initial $V \mathrm{~T}$ of $5 \mathrm{cc} \cdot \mathrm{kg}^{-1}$ or less. With recruitment, the slope of the flow-time curve moves rightward as the lung elastance decreases, thereby accommodating an increase in $V \mathrm{~T}$ for a given pressure (figure 3). Thus, both in practice and experimentally, TCAV traditionally achieves VTs that adapt to lung compliance, as originally intended with the "baby lung" concept, and is personalised, rather than a one-size-fits-all approach [11]. The concept of the baby lung is based on the residual aeratable lung volume which is a near function of compliance rather than body weight [45]. Therefore, normalising $V \mathrm{~T}$ to compliance may prove superior to body weight. As a result of $V$ T normalised to compliance, the driving pressure remains low in APRV [34]. Both driving pressure and exercise physiology data further validate a relationship between compliance and VT size $[46,47]$.

In a computational model of lung mechanics, TCAV with larger $V \mathrm{~T}$ across a compliant lung demonstrated greater recruitment than $L V T$ ventilation yet no increase in tissue overdistension [23]. With the improved alveolar recruitment, stability and homogeneity that TCAV generates, these larger VTs are distributed across a uniform set of open alveoli, thus reducing the alveolar microstrain [22]. Similarly, in a recent study, SiLva et al. [14] showed that despite slightly larger VT, the TCAV group expressed less amphiregulin, a gene expressed during overdistension, as compared with the LVT group [48].

In the porcine extrapulmonary ARDS model, TCAV was associated with significantly greater $P$ plat as compared with LVT, yet the two groups had statistically similar transpulmonary pressure [34]. Thus, the majority of the greater Pplat in APRV was being partitioned towards the chest wall and not towards stressing the lung, leading to an increase in pleural pressure and a reduction in chest wall elastance (figure 4) [34]. It has been established that the respiratory system, particularly in the setting of heterogeneous injury, is made of a gradient of fast to slowly distensible compartments [49, 50]. These "slow" compartments may not represent only the less compliant, dependent lung, but also the chest wall,

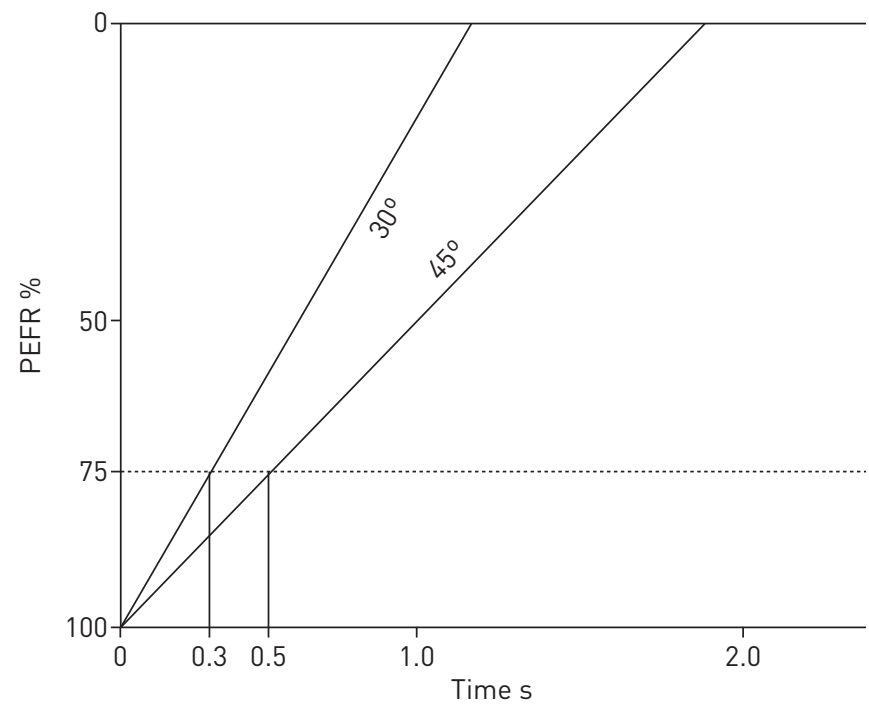

FIGURE 3 Depiction of an expiratory flow curve. A patient with a lung that initially has low compliance has a steeper expiratory flow curve $\left(30^{\circ}\right)$ and will require a short release phase (TLow) $(0.3 \mathrm{~s}$ in this example) to terminate the expiratory flow rate at $75 \%$ of the peak expiratory flow (PEFR). As the lung recruits and becomes more compliant, the slope decreases to $45^{\circ}$, requiring an extension in the TLow time, in this example to $0.5 \mathrm{~s}$. With alveolar recruitment and increasing compliance, the lung is able to accommodate larger tidal volumes. Thus, airway pressure release ventilation allows for mechanical ventilation that is time controlled and adaptive to the patient's respiratory system mechanics (time-controlled adaptive ventilation). Reproduced with permission from the Intensive Care On-line Network (ICON). 

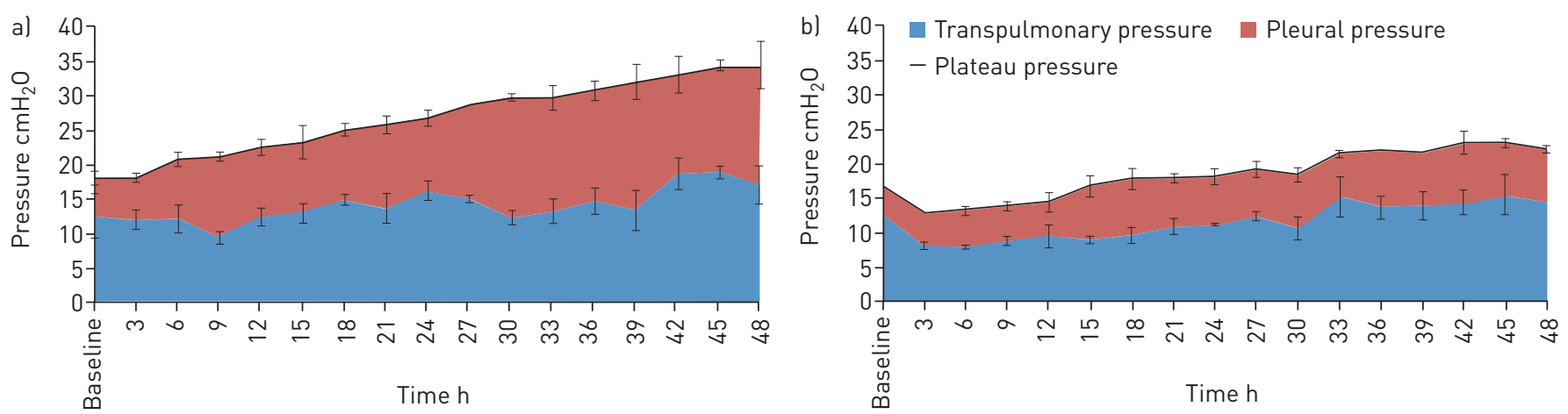

FIGURE 4 In a porcine extrapulmonary acute respiratory distress syndrome model, a) time-controlled adaptive ventilation (TCAV) was compared against b) low tidal volume (LVT). The plateau pressure ( $P$ plat) was partitioned into pleural pressure and transpulmonary pressure. Despite the higher Pplat in the TCAV group, the transpulmonary pressure between TCAV and LVT was similar, as the majority of the pressure was being distributed as pleural pressure. In this study, the TCAV group demonstrated a reduction in respiratory system, chest wall and lung elastance (data not shown). Reproduced and modified from [34] with permission.

which may require additional time at a given pressure, sometimes hours, to recruit [49]. Thus TCAV with an extended time at $P$ plat provides the time to slowly coax open the lung, recruiting the slow compartments, causing stress relaxation of both the lung and chest wall [34, 49, 51].

Although the importance of actively recruiting respiratory muscles during periods of physiological stress has been described in obstructive lung disease [52] and even in playing wind instruments [53], this concept of passively recruiting the chest wall with ventilation is relatively recent. In a vertical direction, providing sufficient pressure to oppose the elevated intra-abdominal pressure associated with extrapulmonary ARDS will shift the volume-pressure curve of the chest back to the left and increase diaphragm tension at end-expiration [54]. This strategy of distributing pressure to the chest wall, as well as a brief expiratory release time to minimise chest wall elastic recoil, represents a novel and exciting mechanism by which TCAV may be protective [34].

\section{Micro-environment}

Minimising lung stress and strain has been recognised as a key mechanism by which VILI might be prevented [55]. Although conceptually this is an intuitive idea, it is not practically easy to measure lung stress and strain. Pplat has been recognised as an inadequate surrogate of lung stress, as the degree of the $P$ plat distributed towards distending or stressing the lung is dependent on the chest wall elastance [56]. Moreover, the whole-lung stress does not account for the alveolar stress-risers [57] that cause heterogeneous inflation of adjacent alveoli and greater alveolar stress $[58,59]$. Thus, a mechanical ventilation strategy with a relatively low whole-lung strain may still have significantly greater microstrain [22]. Even theoretically $\mathrm{L} V \mathrm{~T}$, if delivered to an overestimated "baby lung", may cause regional alveolar overdistension while the less compliant lung remains collapsed $[19,60]$.

Regional stresses and strains may be amplified in the heterogeneous lung at the microscale, such that a delivered volume and pressure (i.e. stress), will be unevenly and preferentially distributed to the interface between normal and atelectatic regions. This uneven distribution produces stress-risers within a cluster of airspaces, causing discordant alveolar expansion (i.e. overdistension) and microstrain (i.e. R/D) [61]. Thus, promoting lung homogeneity with ventilation ensures that a given $V \mathrm{~T}$ will be distributed evenly across the lung, reducing atelectasis and $\mathrm{R} / \mathrm{D}$, and should be a primary goal of ventilation [58]. The time during the expiratory release (TLow) in TCAV is precisely regulated to achieve a ratio of the end expiratory flow (EEF)/peak expiratory flow rate (PEFR) ratio of $75 \%$, as this has been shown to improve oxygenation [9-12, 24] and optimise alveolar stability by preventing end-expiratory collapse [22]. Extending the TLow beyond a EEF/PEFR ratio of $75 \%$ in acute, restrictive lung disease with the use of the APRV mode is not a feature of the TCAV protocol unless the slope of the expiratory flow curve exhibits chronic obstructive lung disease. Extending the TLow causes a positive skew in the alveolar size distributions at expiration (figure 5). Despite this, the alveolar size distributions were similar to that of control at inspiration, regardless of EEF/PEFR ratio, suggesting that the extended time at the $P$ plat is sufficient to recruit alveoli (figure 5) [59]. Thus, if the TLow is set longer than the collapse time of the unstable alveoli, or the expiratory pressure reached at the end of Tlow is lower than the closing pressure of the unstable alveoli, the alveoli will tend to collapse, leading to repetitive R/D with each tidal breath and increased microstrain [22]. The change in alveolar size distribution between inspiration and expiration is termed dynamic heterogeneity where dynamic heterogeneity indicates pathological $\mathrm{R} / \mathrm{D}$ 

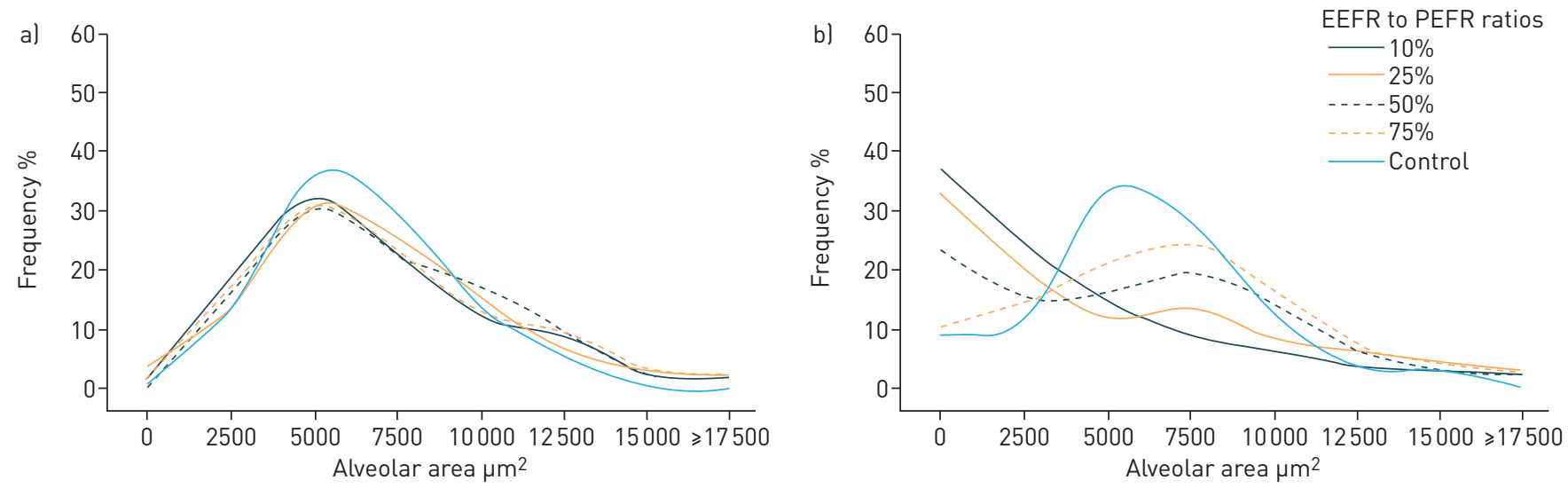

FIGURE 5 In an in vivo microscopy rat study of subpleural alveoli, normal healthy lungs (control) were compared with airway pressure release ventilation (APRV) in a pulmonary acute respiratory distress syndrome model. al Time-controlled adaptive ventilation (TCAV) versus control (inspiration); b) TCAV versus control (expiration). The end-expiratory flow (EEF) rate/peak expiratory flow rate (PEFR) ratio was varied to demonstrate the importance of setting APRV with an appropriate release phase (TLow) (to maintain EEF/PEFR at 75\% as described in the TCAV protocol). Regardless of the TLow, there is an alveolar size distribution similar to that of the control group at inspiration, in spite of the lung injury, suggesting the extended time at $P$ High is sufficient to maintain alveolar recruitment. However, lengthening the TLow such that the EEF/PEFR ratio is $75 \%$ creates a positive skew with alveolar collapse and instability. Reproduced and modified from [59] with permission.

with each tidal breath. With an EEF/PEFR ratio of $75 \%$, there was dynamic homogeneity between inspiration and expiration with the mean alveolar size at inspiration closely approximating the mean alveolar size at expiration [59]. Therefore, just as increasing PEEP leads to alveolar stabilisation at end-expiration, the expiratory release time of APRV may be modified by precisely regulating the EEF/ PEFR ratio to $75 \%$, in accordance with the TCAV protocol $[62,63]$.

As established previously, TCAV has been associated with larger $V \mathrm{~T}$ (when lung compliance is normal and the lung is fully recruited); however, this does not necessarily imply there is greater lung strain. In a study evaluating the relationship between lung strain and alveolar micro-strain using in vivo microscopy, it was found that TCAV set appropriately (with an EEF/PEFR ratio of 75\%) had a significantly lower alveolar microstrain despite higher macrostrain. By comparison, the lung strain and alveolar microstrain in animals ventilated with $V \mathrm{~T}$ of $6 \mathrm{cc} \cdot \mathrm{kg}^{-1}$ and PEEP $16 \mathrm{cmH}_{2} \mathrm{O}$ was similar to that of TCAV, but with less alveolar recruitment [22]. Animals ventilated with $V \mathrm{~T}$ of $6 \mathrm{cc} \cdot \mathrm{kg}^{-1}$ and PEEP $5 \mathrm{cmH}_{2} \mathrm{O}$ had a relatively low lung strain yet a significantly higher alveolar microstrain, confirming that even low $V \mathrm{~T}$ may be injurious if the "baby lung" is small enough $[22,60]$. This study also illustrated the importance of setting APRV with an appropriate EEF/PEFR ratio, as an EEF/PEFR ratio of $10 \%$ led to significantly greater alveolar microstrain and R/D as compared with an EEF/PEFR ratio of $75 \%[22,23]$. With an EEF/PEFR ratio of $10 \%$, the lung $V \mathrm{~T}$ are significantly greater, as the lung is allowed additional time to fall below its intrinsic functional residual capacity (FRC) during the expiratory release phase, following which these larger VTs are then distributed across an unstable, heterogeneous lung [22, 23]. In addition, this in vivo microscopy study demonstrated the difference between lung $V \mathrm{~T}$ (the volume driven into the respiratory system by the ventilator and measured at the level of the trachea) and alveolar $V_{T}$ (the degree to which the alveoli distend in response to the tracheal $V \mathrm{~T}$ ). In a collapsed, heterogeneous lung, even a small lung $V \mathrm{~T}$ may lead to large alveolar $V \mathrm{~T}$, as observed in the low-PEEP groups with $6 \mathrm{cc} \cdot \mathrm{kg}^{-1} V \mathrm{~T}$, but in a fully recruited and homogenous lung, a large $V \mathrm{~T}$ may be administered and lead to low alveolar $V \mathrm{~T}$, as observed in the APRV group set according to the TCAV protocol [22].

In a markedly injured lung with significantly reduced alveolar compliance, $V T$ may be preferentially distributed to the alveolar ducts and conducting airways instead of the alveoli. In a parallel study, it was determined that TCAV with an EEF/PEFR ratio of $75 \%$ minimised conducting airway microstrain and maximised alveolar air space occupancy compared with the other experimental settings, including APRV with an EEF/PEFR ratio of $10 \%$, LVT with PEEP $5 \mathrm{cmH}_{2} \mathrm{O}$ and PEEP $16 \mathrm{cmH}_{2} \mathrm{O}$. This study suggests that in those same settings with reduced alveolar recruitment and stability, the majority of the tidal breath was being accommodated by the more compliant conducting airways [21].

\section{Spontaneous breathing and cardiopulmonary effects}

The lung expands homogenously when it nears total lung capacity (TLC) [64] from FRC, thus inspiratory pressures (CPAP phase) in TCAV are conventionally set to obtain lung volumes on the steep portion of the pressure-volume curve; above FRC, but less than TLC. Inspiration from volumes above FRC, 40\% of the vital capacity, distribute ventilation predominantly to the basilar lung regions [65] promoting alveolar 
recruitment while avoiding overdistension and encouraging spontaneous breathing by patients at any phase of the respiratory cycle $[15,66,67]$. Thus, the CPAP phase of TCAV decreases the work of breathing performed by the patient by increasing the lung volume and compliance $[66,68-70]$ and can be used in patients requiring partial or full ventilator support [71, 72]. CPAP decreases the inspiratory work of breathing and sense of breathlessness in patients with severe chronic obstructive pulmonary disease (COPD) and acute respiratory failure by reducing the inspiratory mechanical load [73]. Furthermore, CPAP decreases dynamic hyperinflation in patients with COPD, thus further decreasing the work of breathing [74].

Although patients do not need to be breathing spontaneously in order to use the TCAV protocol for mechanical ventilation, spontaneous breathing with TCAV provides the benefit of increasing end-expiratory lung volume, decreasing atelectasis and improving distribution of ventilation to dependent lung regions [75-77]. Although spontaneous breathing causes a decrease in intrathoracic pressure and usually increases transpulmonary pressure, it has been demonstrated that spontaneous breathing in TCAV actually causes a reduction in transpulmonary pressure [78], promoting instead regional increases in transpulmonary pressure to recruit dependent lung regions [79]. Higher PEEP levels have been shown to render spontaneous breathing non-injurious and decreases air hunger through the effect on pulmonary stretch receptors limiting high inspiratory efforts [69, 80, 81]. Similarly, the higher end-expiratory lung volume allows the patient to begin a spontaneous breath at a more favourable lung volume (FRC) and limits the ability of the diaphragm to generate high transpulmonary pressure [74]. However, spontaneous ventilation can be injurious with high inspiratory effort at a low lung volume or low PEEP [82]. Spontaneous breathing in TCAV largely occurs at the upper CPAP level ( $90 \%$ of the cycle time), unlike $\mathrm{LVT}$ ventilation in which the majority of spontaneous breathing occurs at the lower PEEP level. Furthermore, the ability of the patient to exhale at any time through an open expiratory valve [67] enables the patient to defend their lung volume and prevent overdistension and dynamic hyperinflation [83-85].

Maintaining spontaneous breathing with TCAV has been associated with reduced requirements for sedation and neuromuscular blockade, demonstrating that this is a comfortable mode for patients [20, 67, 86, 87]. Promoting patient comfort and spontaneous breathing allows for further chest wall and lung recruitment, translating into a reduction in negative clinical outcomes. For instance, patients with pulmonary contusions placed on APRV as compared with a conventional mode of ventilation reduced ventilator-associated pneumonia, despite having a greater lung injury score [88]. Spontaneous breathing offers the potential to be a diaphragm-protective ventilator strategy, limiting ventilator-induced diaphragm dysfunction with an improvement in outcomes [89].

In addition to the positive effects on pulmonary function, TCAV in combination with spontaneous breathing has been associated with an improved haemodynamic profile. Spontaneous breathing decreases intrathoracic pressure, allowing for improved venous return to the heart, an increase in preload and cardiac output $[67,72,75,78,79]$. Therefore, spontaneous breathing in TCAV has demonstrated improvement in systemic blood flow [90], perfusion of the preportal organs [90, 91], kidneys [92], cerebrum and spinal cord [93]. This has led to an improvement in renal function [92], increase in urine output, decrease in lactate and decreased pressor requirement [67]. Furthermore, TCAV has demonstrated an improvement in lung perfusion in children following cardiac surgery [94] and spontaneous breathing has been shown to further improve blood flow to dependent lung regions in TCAV [76]. The reduced dead space achieved by recruiting the dependent lung region [72] combined with the improved blood flow to this region therefore reduces ventilator-perfusion mismatch and improves oxygenation [77].

\section{APRV critique}

Although some articles have claimed there is an increased work of breathing or asynchrony when setting APRV [95-97], it is important to recognise that these studies did not utilise the TCAV protocol and used ventilators that did not offer APRV as a mode (instead requiring adaptive modules to mimic APRV). Theoretically, assisted breathing can be added to APRV, but it is unnecessary and changes the architecture of a spontaneous breath from sinusoidal to decelerating, where the flow and pressure are uncoupled from patient effort. In essence, APRV set with the TCAV protocol is CPAP with a release phase and the TCAV protocol advocates against attempting synchronisation with assisted breathing. Other studies that have failed to demonstrate beneficial results with APRV have set respiratory rates rather than modifying THigh and TLow according to respiratory physiology [96], set a $P$ Low of $0 \mathrm{cmH}_{2} \mathrm{O}$, but set the TLow long enough that the lung is allowed to collapse back down to below functional residual capacity with each ventilator cycle [98], or have set $P_{\text {Low }}>0 \mathrm{cmH}_{2} \mathrm{O}$ rather than using the TLow to modify the end-expiratory lung volume [96]. These examples serve to highlight that when evaluating studies of APRV it is critical to investigate the ventilator settings applied, as this may alter the interpretation of the results.

The expiratory flow curve is determined by the patient's underlying lung and pathology, therefore making dynamic adjustments to the ventilator parameters based on the expiratory flow allows the ventilator to be 
tailored to the lung and disease process. One of the premises behind TCAV is that it adapts to the changing lung physiology by analysing the expiratory flow curve, thereby providing more support to the injured lung and relaxing support on the recovering lung. For instance, if the TCAV protocol is applied to a patient with increased lung elastance, the release volume will decrease proportionally. Over time, the extended duration at $P$ High will recruit the lung such that the elastance and generated $V$ T will increase accordingly. Thus, the $V \mathrm{~T}$ in the TCAV protocol is personalised and adaptive. One randomised controlled trial [99] sought to impose LVT $\left(6-7 \mathrm{cc} \cdot \mathrm{kg}^{-1}\right)$ to patients on APRV (APRV-LVT) and found that there was a higher mortality as compared with standard LVT ventilation Another three-armed study [100] aimed at comparing $\mathrm{L} V \mathrm{~T}$ with APRV-LVT and TCAV revealed no significant difference between the three groups in terms of oxygenation or mortality, with the main flaw of the study being that it was limited by power. In addition, the authors reported concerns regarding measured release volumes of $>8 \mathrm{cc} \cdot \mathrm{kg}^{-1}$ in the APRV and APRV-LVT groups, but made no mention of the driving pressure or compliance [100]. In an open, compliant lung, this $V \mathrm{~T}$ is probably affiliated with a low driving pressure and will be distributed across a set of open homogenous alveoli reflecting small alveolar $V_{\mathrm{T}}$ [22]. Restricting APRV to generate a specific $V \mathrm{~T}$ goes against the fundamental principles of the TCAV protocol, which allows the changes in lung physiology to determine the release volume generated.

\section{Conclusion}

The TCAV protocol using the APRV mode has demonstrated efficacy in a prospective review of intensive care unit patients [20], a retrospective review of severely injured trauma patients [8], a porcine sepsis and gut ischaemia/reperfusion-induced ARDS model [9, 11, 34], a rat VILI [12], and rat pulmonary and extrapulmonary ARDS models [10, 14]. The extended time at Pplat lends itself to the hypothesis that TCAV is able to recruit alveoli, thereby increasing the alveolar surface area available for gas exchange. The brief release phase minimises derecruitment and provides real-time respiratory system elastance monitoring, harmonising $V \mathrm{~T}$ titration to evolving lung compliance rather than body weight. The combination of recent experimental and clinical evidence demonstrates that TCAV not only does this, but also minimises alveolar heterogeneity and microstrain, conducting airway microstrain, and potentially recruits the chest wall in extrapulmonary ARDS. Although there are limitations to the clinical studies of TCAV with ZHOU et al.'s [20] prospective review not being blinded and ANDREws et al.'s [8] retrospective review being exclusive to trauma patients, where trauma patients carry a specific phenotype of ARDS [101], and randomised controlled trials are needed to test the efficacy of the TCAV protocol further [13], this review of experimental evidence demonstrates that TCAV may be effective in preventing VILI and ARDS by a pleiotropy of mechanisms.

Author contributions: The authors maintain that industry had no role in the design and conduct of the study; the collection, management, analysis, or interpretation of the data; or the preparation, review, or approval of the manuscript.

Conflict of interest: M. Kollisch-Singule reports grants from NIH during the conduct of the study, and other funding from Dräger Medical Systems, Inc. and the Intensive Care On-line Network, Inc. (ICON), outside the submitted work. P. Andrews has presented and received honoraria and travel reimbursement at events sponsored by Dräger Medical outside of the submitted work. She is employed by the Intensive Care On-line Network (ICON). J. Satalin has nothing to disclose. L.A. Gatto reports being listed as co-inventor in U.S. Patent No. 5977091 issued to the Research Foundation of SUNY (no related funds or royalties). G.F. Nieman reports grants from Drager Medical, outside the submitted work. In addition, He has patents issued on the method of preventing acute lung injury, the method of treating sepsis-induced ARDS, the novel method of assessing alveolar inflation, the method of reducing blood loss associated with cardiopulmonary bypass, minimally invasive suction and treatment device (MIST), and apparatus system and method for assessing alveolar inflation issued. N.M. Habashi has presented and received honoraria and travel reimbursement at events sponsored by Dräger Medical outside of the submitted work. He holds patents in the area of mechanical ventilation and is the founder of the Intensive Care On-line Network (ICON).

Supporting statement: Funding was received from the U.S. Department of Health and Human Services, National Institutes of Health, National Heart, Lung, and Blood Institute (R01 HL131143). Funding information for this article has been deposited with the Crossref Funder Registry.

\section{References}

1 Rubenfeld GD, Caldwell E, Peabody E, et al. Incidence and outcomes of acute lung injury. N Engl J Med 2005; 353: $1685-1693$.

2 Villar J, Blanco J, Añón JM, et al. The ALIEN study: incidence and outcome of acute respiratory distress syndrome in the era of lung protective ventilation. Intensive Care Med 2011; 37: 1932-1941.

Ware LB, Matthay MA. The acute respiratory distress syndrome. N Engl J Med 2000; 342: 1334-1349.

Bellani G, Laffey JG, Pham T, et al. The LUNG SAFE study: a presentation of the prevalence of ARDS according to the Berlin Definition! Crit Care 2016; 20: 268.

5 Amato MB, Barbas CS, Medeiros DM, et al. Effect of a protective-ventilation strategy on mortality in the acute respiratory distress syndrome. $N$ Engl J Med 1998; 338: 347-354.

6 ARDSnet. NIH NHLBI ARDS Clinical Network Mechanical Ventilation Protocol Summary. ARDSnet, 2000. 
Gupta S, Joshi V, Joshi P, et al. Airway pressure release ventilation: a neonatal case series and review of current practice. Can Respir J 2013; 20: e86-e91.

Andrews P, Shiber J, Jaruga-Killeen E, et al. Early application of airway pressure release ventilation may reduce mortality in high-risk trauma patients: a systematic review of observational trauma ARDS literature. $J$ Trauma Acute Care Surg 2013; 75: 635-641.

Roy S, Sadowitz B, Andrews P, et al. Early stabilizing alveolar ventilation prevents acute respiratory distress syndrome: a novel timing-based ventilatory intervention to avert lung injury. J Trauma Acute Care Surg 2012; 73: 391-400.

Roy SK, Emr B, Sadowitz B, et al. Preemptive application of airway pressure release ventilation prevents development of acute respiratory distress syndrome in a rat traumatic hemorrhagic shock model. Shock 2013; 40: 210-216.

Roy S, Habashi N, Sadowitz B, et al. Early airway pressure release ventilation prevents ARDS - a novel preventive approach to lung injury. Shock 2013; 39: 28-38.

Emr B, Gatto L, Roy S, et al. Airway pressure release ventilation prevents ventilator-induced lung injury in normal lungs. JAMA Surg 2013; 148: 1005-1012.

Mireles-Cabodevila E, Kacmarek RM. Should airway pressure release ventilation be the primary mode in ARDS? Respir Care 2016; 61: 761-773.

Silva PL, Cruz FF, Samary CDS, et al. Biological response to time-controlled adaptive ventilation depends on acute respiratory distress syndrome etiology. Crit Care Med 2018; 46: e609-e617.

Habashi NM. Other approaches to open-lung ventilation: airway pressure release ventilation. Crit Care Med 2005, 33: 3 Suppl., S228-S240.

Guttmann J, Eberhard L, Fabry B, et al. Time constant/volume relationship of passive expiration in mechanically ventilated ARDS patients. Eur Respir J 1995; 8: 114-120.

Ashutosh K, Keighley JF. Passive expiration as a test of lung function. Thorax 1978; 33: 740-746.

Chelucci GL, Dall'Ava-Santucci J, Dhainaut JF, et al. Association of PEEP with two different inflation volumes in ARDS patients: effects on passive lung deflation and alveolar recruitment. Intensive Care Med 2000; 26: 870-877.

Kollisch-Singule MC, Jain SV, Andrews PL, et al. Looking beyond macroventilatory parameters and rethinking ventilator-induced lung injury. J Appl Physiol 2018; 124: 1214-1218.

Zhou Y, Jin X, Lv Y, et al. Early application of airway pressure release ventilation may reduce the duration of mechanical ventilation in acute respiratory distress syndrome. Intensive Care Med 2017; 43: 1648-1659.

Kollisch-Singule M, Emr B, Smith B, et al. Airway pressure release ventilation reduces conducting airway micro-strain in lung injury. J Am Coll Surg 2014; 219: 968-976.

Kollisch-Singule M, Emr B, Smith B, et al. Mechanical breath profile of airway pressure release ventilation: the effect on alveolar recruitment and microstrain in acute lung injury. JAMA Surg 2014; 149: 1138-1145.

Smith BJ, Lundblad LK, Kollisch-Singule M, et al. Predicting the response of the injured lung to the mechanical breath profile. J Appl Physiol 2015; 118: 932-940. respiratory distress syndrome. Exp Ther Med 2017; 14: 1941-1946.

Stenqvist O. Practical assessment of respiratory mechanics. Br J Anaesth 2003; 91: 92-105.

Albert SP, DiRocco J, Allen GB, et al. The role of time and pressure on alveolar recruitment. J Appl Physiol 2009; 106: $757-765$

Lachmann B. Open up the lung and keep the lung open. Intensive Care Med 1992; 18: 319-321.

Bates JH, Irvin CG. Time dependence of recruitment and derecruitment in the lung: a theoretical model. J Appl Physiol 2002; 93: 705-713.

Motta-Ribeiro GC, Hashimoto S, Winkler T, et al. Deterioration of regional lung strain and inflammation during early lung injury. Am J Respir Crit Care Med 2018; 198: 891-902.

Gattinoni L, Tonetti T, Cressoni $\mathrm{M}$, et al. Ventilator-related causes of lung injury: the mechanical power. Intensive Care Med 2016; 42: 1567-1575.

Albert RK, Lakshminarayan S, Hildebrandt J, et al. Increased surface tension favors pulmonary edema formation in anesthetized dogs' lungs. J Clin Invest 1979; 63: 1015-1018.

Dreyfuss D, Basset G, Soler P, et al. Intermittent positive-pressure hyperventilation with high inflation pressures produces pulmonary microvascular injury in rats. Am Rev Respir Dis 1985; 132: 880-884.

Parker JC, Townsley MI, Rippe B, et al. Increased microvascular permeability in dog lungs due to high peak airway pressures. J Appl Physiol Respir Environ Exerc Physiol 1984; 57: 1809-1816.

Kollisch-Singule M, Emr B, Jain SV, et al. The effects of airway pressure release ventilation on respiratory mechanics in extrapulmonary lung injury. Intensive Care Med Exp 2015; 3: 35.

Tremblay L, Valenza F, Ribeiro SP, et al. Injurious ventilatory strategies increase cytokines and c-fos m-RNA expression in an isolated rat lung model. J Clin Invest 1997; 99: 944-952.

Schwartz MD, Moore EE, Moore FA, et al. Nuclear factor-kappa B is activated in alveolar macrophages from patients with acute respiratory distress syndrome. Crit Care Med 1996; 24: 1285-1292.

Imai Y, Parodo J, Kajikawa O, et al. Injurious mechanical ventilation and end-organ epithelial cell apoptosis and organ dysfunction in an experimental model of acute respiratory distress syndrome. JAMA 2003; 289: 2104-2112.

Montgomery AB, Stager MA, Spatola R, et al. Causes of mortality in patients with the adult respiratory distress syndrome. Am Rev Respir Dis 1985; 132: 485-489.

Borelli M, Kolobow T, Spatola R, et al. Severe acute respiratory failure managed with continuous positive airway pressure and partial extracorporeal carbon dioxide removal by an artificial membrane lung. A controlled, randomized animal study. Am Rev Respir Dis 1988; 138: 1480-1487.

Ranieri VM, Suter PM, Tortorella C, et al. Effect of mechanical ventilation on inflammatory mediators in patients with acute respiratory distress syndrome: a randomized controlled trial. JAMA 1999; 282: 54-61.

Slutsky AS, Tremblay LN. Multiple system organ failure. Is mechanical ventilation a contributing factor? Am J Respir Crit Care Med 1998; 157: 1721-1725.

Albert S, Kubiak BD, Vieau CJ, et al. Comparison of "open lung" modes with low tidal volumes in a porcine lung injury model. J Surg Res 2011; 166: e71-e81. 
Douzinas EE, Tsidemiadou PD, Pitaridis MT, et al. The regional production of cytokines and lactate in sepsis-related multiple organ failure. Am J Respir Crit Care Med 1997; 155: 53-59.

Man Y, Hart VJ, Ring CJ, et al. Loss of epithelial integrity resulting from E-cadherin dysfunction predisposes airway epithelial cells to adenoviral infection. Am J Respir Cell Mol Biol 2000; 23: 610-617.

Gattinoni L, Pesenti A. The concept of "baby lung". In: Pinsky MR, Brochard L, Mancebo J, eds. Applied Physiology in Intensive Care Medicine. Berlin Heidelberg, Springer-Verlag, 2006; pp. 303-309.

Amato MB, Meade MO, Slutsky AS, et al. Driving pressure and survival in the acute respiratory distress syndrome. N Engl J Med 2015; 372: 747-755.

Chiang ST. Distribution of ventilation and frequency-dependence of dynamic lung compliance. Thorax 1971; 26 : 721-726.

Dolinay T, Kaminski N, Felgendreher M, et al. Gene expression profiling of target genes in ventilator-induced lung injury. Physiol Genomics 2006; 26: 68-75.

Fretschner R, Laubscher TP, Brunner JX. New aspects of pulmonary mechanics: "slowly" distensible compartments of the respiratory system, identified by a PEEP step maneuver. Intensive Care Med 1996; 22: $1328-1334$.

Stenqvist O, Grivans C, Andersson B, et al. Lung elastance and transpulmonary pressure can be determined without using oesophageal pressure measurements. Acta Anaesthesiol Scand 2012; 56: 738-747.

Katz JA, Ozanne GM, Zinn SE, et al. Time course and mechanisms of lung-volume increase with PEEP in acute pulmonary failure. Anesthesiology 1981; 54: 9-16.

Breslin EH. The pattern of respiratory muscle recruitment during pursed-lip breathing. Chest 1992; 101: 75-78. Cossette I, Monaco P, Aliverti A, et al. Chest wall dynamics and muscle recruitment during professional flute playing. Respir Physiol Neurobiol 2008; 160: 187-195.

Lundin S, Grivans C, Stenqvist O. Transpulmonary pressure and lung elastance can be estimated by a PEEP-step manoeuvre. Acta Anaesthesiol Scand 2015; 59: 185-196.

Protti A, Andreis DT, Monti M, et al. Lung stress and strain during mechanical ventilation: any difference between statics and dynamics? Crit Care Med 2013; 41: 1046-1055.

Chiumello D, Carlesso E, Cadringher $\mathrm{P}$, et al. Lung stress and strain during mechanical ventilation for acute respiratory distress syndrome. Am J Respir Crit Care Med 2008; 178: 346-355.

Mead J, Takishima T, Leith D. Stress distribution in lungs: a model of pulmonary elasticity. J Appl Physiol 1970; 28: 596-608.

Cressoni M, Cadringher P, Chiurazzi C, et al. Lung inhomogeneity in patients with acute respiratory distress syndrome. Am J Respir Crit Care Med 2014; 189: 149-158.

Kollisch-Singule M, Jain S, Andrews $\mathrm{P}$, et al. Effect of airway pressure release ventilation on dynamic alveolar heterogeneity. JAMA Surg 2016; 151: 64-72.

Terragni PP, Rosboch G, Tealdi A, et al. Tidal hyperinflation during low tidal volume ventilation in acute respiratory distress syndrome. Am J Respir Crit Care Med 2007; 175: 160-166.

Xin Y, Cereda M, Hamedani $\mathrm{H}$, et al. Unstable inflation causing injury: insight from prone position and paired computed tomography scans. Am J Respir Crit Care Med 2018; 198: 197-207. recruitment? Am J Respir Cell Mol Biol 2008; 38: 572-578.

Mazzuca E, Salito C, Rivolta I, et al. From morphological heterogeneity at alveolar level to the overall mechanical lung behavior: an in vivo microscopic imaging study. Physiol Rep 2014; 2: e00221.

Chevalier PA, Rodarte JR, Harris LD. Regional lung expansion at total lung capacity in intact $v s$. excised canine lungs. I Appl Physiol Respir Environ Exerc Physiol 1978; 45: 363-369.

Robertson PC, Anthonisen NR, Ross D. Effect of inspiratory flow rate on regional distribution of inspired gas. J Appl Physiol 1969; 26: 438-443.

Downs JB, Stock MC. Airway pressure release ventilation: a new concept in ventilatory support. Crit Care Med 1987; 15: 459-461.

Kaplan LJ, Bailey H, Formosa V. Airway pressure release ventilation increases cardiac performance in patients with acute lung injury/adult respiratory distress syndrome. Crit Care 2001; 5: 221-226.

Hering R, Zinserling J, Wrigge $\mathrm{H}$, et al. Effects of spontaneous breathing during airway pressure release ventilation on respiratory work and muscle blood flow in experimental lung injury. Chest 2005; 128: 2991-2998.

Vovk A, Binks AP. Raising end-expiratory volume relieves air hunger in mechanically ventilated healthy adults. J Appl Physiol 2007; 103: 779-786.

Smith TC, Marini JJ. Impact of PEEP on lung mechanics and work of breathing in severe airflow obstruction. J Appl Physiol 1988; 65: 1488-1499.

Henzler D. What on earth is APRV? Crit Care 2011; 15: 115.

Putensen C, Mutz NJ, Putensen-Himmer G, et al. Spontaneous breathing during ventilatory support improves ventilation-perfusion distributions in patients with acute respiratory distress syndrome. Am J Respir Crit Care Med 1999; 159: 1241-1248.

Petrof BJ, Legaré M, Goldberg P, et al. Continuous positive airway pressure reduces work of breathing and dyspnea during weaning from mechanical ventilation in severe chronic obstructive pulmonary disease. Am Rev Respir Dis 1990; 141: 281-289.

O’Donoghue FJ, Catcheside PG, Jordan AS, et al. Effect of CPAP on intrinsic PEEP, inspiratory effort, and lung volume in severe stable COPD. Thorax 2002; 57: 533-539.

Wrigge H, Zinserling J, Neumann P, et al. Spontaneous breathing improves lung aeration in oleic acid-induced lung injury. Anesthesiology 2003; 99: 376-384.

Neumann $\mathrm{P}$, Wrigge $\mathrm{H}$, Zinserling J, et al. Spontaneous breathing affects the spatial ventilation and perfusion distribution during mechanical ventilatory support. Crit Care Med 2005; 33: 1090-1095.

Wrigge H, Zinserling J, Neumann P, et al. Spontaneous breathing with airway pressure release ventilation favors ventilation in dependent lung regions and counters cyclic alveolar collapse in oleic-acid-induced lung injury: a randomized controlled computed tomography trial. Crit Care 2005; 9: R780-R789.

Räsänen J, Downs JB, Stock MC. Cardiovascular effects of conventional positive pressure ventilation and airway pressure release ventilation. Chest 1988; 93: 911-915. 
Henzler D, Dembinski R, Bensberg R, et al. Ventilation with biphasic positive airway pressure in experimental lung injury. Influence of transpulmonary pressure on gas exchange and haemodynamics. Intensive Care Med 2004; 30: 935-943.

Morais CCA, Koyama Y, Yoshida T, et al. High positive end-expiratory pressure renders spontaneous effort noninjurious. Am J Respir Crit Care Med 2018; 197: 1285-1296.

Schlobohm RM, Falltrick RT, Quan SF, et al. Lung volumes, mechanics, and oxygenation during spontaneous positive-pressure ventilation: the advantage of CPAP over EPAP. Anesthesiology 1981; 55: 416-422.

less positive end-expiratory pressure. Crit Care Med 2016; 44: e678-e688.

Road JD, Leevers AM, Goldman E, et al. Respiratory muscle coordination and diaphragm length during expiratory threshold loading. J Appl Physiol 1991; 70: 1554-1562.

Leevers AM, Road JD. Mechanical response to hyperinflation of the two abdominal muscle layers. J Appl Physiol 1989; 66: 2189-2195.

Farkas GA, Schroeder MA. Mechanical role of expiratory muscles during breathing in prone anesthetized dogs. J Appl Physiol 1990; 69: 2137-2142.

Putensen $\mathrm{C}$, Zech S, Wrigge $\mathrm{H}$, et al. Long-term effects of spontaneous breathing during ventilatory support in patients with acute lung injury. Am J Respir Crit Care Med 2001; 164: 43-49.

Rathgeber J, Schorn B, Falk V, et al. The influence of controlled mandatory ventilation (CMV), intermittent mandatory ventilation (IMV) and biphasic intermittent positive airway pressure (BIPAP) on duration of intubation and consumption of analgesics and sedatives. A prospective analysis in 596 patients following adult cardiac surgery. Eur J Anaesthesiol 1997; 14: 576-582.

Walkey AJ, Nair S, Papadopoulos S, et al. Use of airway pressure release ventilation is associated with a reduced incidence of ventilator-associated pneumonia in patients with pulmonary contusion. J Trauma 2011; 70: E42-E47.

Goligher EC, Dres M, Fan E, et al. Mechanical ventilation-induced diaphragm atrophy strongly impacts clinical outcomes. Am J Respir Crit Care Med 2018; 197: 204-213.

Hering R, Viehöfer A, Zinserling J, et al. Effects of spontaneous breathing during airway pressure release ventilation on intestinal blood flow in experimental lung injury. Anesthesiology 2003; 99: 1137-1144.

Hering R, Bolten JC, Kreyer S, et al. Spontaneous breathing during airway pressure release ventilation in experimental lung injury: effects on hepatic blood flow. Intensive Care Med 2008; 34: 523-527.

Hering R, Peters D, Zinserling J, et al. Effects of spontaneous breathing during airway pressure release ventilation on renal perfusion and function in patients with acute lung injury. Intensive Care Med 2002; 28: 1426-1433.

Kreyer S, Putensen C, Berg A, et al. Effects of spontaneous breathing during airway pressure release ventilation on cerebral and spinal cord perfusion in experimental acute lung injury. J Neurosurg Anesthesiol 2010; 22: 323-329.

Walsh MA, Merat M, La Rotta G, et al. Airway pressure release ventilation improves pulmonary blood flow in infants after cardiac surgery. Crit Care Med 2011; 39: 2599-2604.

Chiang AA, Steinfeld A, Gropper C, et al. Demand-flow airway pressure release ventilation as a partial ventilatory support mode: comparison with synchronized intermittent mandatory ventilation and pressure support ventilation. Crit Care Med 1994; 22: 1431-1437.

Henzler D, Pelosi P, Bensberg R, et al. Effects of partial ventilatory support modalities on respiratory function in severe hypoxemic lung injury. Crit Care Med 2006; 34: 1738-1745.

Calzia E, Lindner KH, Witt S, et al. Pressure-time product and work of breathing during biphasic continuous positive airway pressure and assisted spontaneous breathing. Am J Respir Crit Care Med 1994; 150: 904-910.

Varpula T, Valta P, Niemi R, et al. Airway pressure release ventilation as a primary ventilatory mode in acute respiratory distress syndrome. Acta Anaesthesiol Scand 2004; 48: 722-731.

Lalgudi Ganesan S, Jayashree M, Chandra Singhi S, et al. Airway pressure release ventilation in pediatric acute respiratory distress syndrome. a randomized controlled trial. Am J Respir Crit Care Med 2018; 198: 1199-1207.

Hirshberg EL, Lanspa MJ, Peterson J, et al. Randomized feasibility trial of a low tidal volume-airway pressure release ventilation protocol compared with traditional airway pressure release ventilation and volume control ventilation protocols. Crit Care Med 2018; 46: 1943-1952.

Calfee CS, Eisner MD, Ware LB, et al. Trauma-associated lung injury differs clinically and biologically from acute lung injury due to other clinical disorders. Crit Care Med 2007; 35: 2243-2250. 